\title{
Risk of venous thromboembolism in people with lung cancer: a cohort study using linked UK healthcare data
}

Alex J Walker, David R Baldwin, Tim R Card, Helen A Powell, Richard B Hubbard and Matthew J Grainge

Correction to: British Journal of Cancer (2016) 115, 115-121. doi:10.1038/bjc.2016.143; Published online 2 June 2016

The authors of the above paper have recognised that they unintentionally omitted information about their funding sources from their paper. The funding statement is included below. The authors apologise for this oversight.

This study was funded by a Population Research Committee project grant from Cancer Research UK (ref: C17683/A12079). 